\title{
Origins of Surgical Robotics: From Space to the Operating Room
}

\author{
Árpád Takács, Dénes Á. Nagy, Imre J. Rudas, \\ Tamás Haidegger
}

Antal Bejczy Center for Intelligent Robotics, Óbuda University, Bécsi út 96/b, H-1034 Budapest, Hungary

\{arpad.takacs, denes.nagy, haidegger, rudas\}@irob.uni-obuda.hu

\begin{abstract}
The rapid development of telerobotic systems led to novel applications beyond the nuclear and industrial domains. Medical telerobotics enabled surgeons to perform medical operations from remote places, far from their patient. Telesurgery systems allow great flexibility, improved performance in general, and support the creation of ideal surgical conditions. The first attempts to develop telesurgical systems borrowed the idea from space research, where the need of novel robots emerged for invasive treatment, even under extreme situations, such as weightlessness. Telesurgical instruments on Earth appeared following the same concept, aiming first for military, then onward for civilian applications. Today, more than 1.5 million patients are receiving telerobotic treatment annually, worldwide. As the surgical robotics domain grew from the initial concepts, it developed along three major concepts: telesurgery, cooperatively controlled robots and automated (image-guided) applications. These domains continue to develop into application specific systems with the goal of reaching the specificity and versatility of conventional surgical instruments.
\end{abstract}

Keywords: surgical robotics; space robotics; teleoperation

\section{Introduction}

In recent years, a large number of surgical robots and robotic surgery related research projects have been introduced and conducted. As a result, useful software and hardware tools appeared on the market, which accelerated the pursuit for new research results in modern robotic surgery and telesurgery [1,2]. Computer-IntegratedSurgery (CIS) and telemedicine are becoming popular in the world's developed countries, improving the quality of medical treatment and patient care. The development of these systems requires a strict and effective cooperation of surgeons, IT experts, engineers and scientists from the various fields of natural and human sciences, creating the possibility of remote or even transcontinental surgery. The concept of these systems often originate from specific extreme applications, thus their 
testing also requires extreme environments, such as weightlessness or extremely high pressure [3].

\section{The Birth of Surgical Robotics}

There is no consensus about the title of "the first surgical robot", since it is hard to define, what criteria should be used to claim a robots role in a surgical procedure fundamental. Generally speaking, the first systems, which appeared in the 1970s were used for different purposes, primarily as assisting devices and supporting manipulators [4]. The concept of telerobotics for surgery appeared in the early 1970s, initiated by the National Aeronautics and Space Administration (NASA) [5]. The goal of the original project was to provide medical assistance for astronauts during their remote mission. For this purpose, remotely controlled robots would have been used, operated from the Earth, as shown in Fig. 1. At that time the proposal was not funded, only limited documentation remained accessible. Although the idea was concluded in a short period of time, another 15 years passed until the first prototypes appeared, mostly backed by the US military. During the development phase, it became apparent that controlling telesurgical robots is very challenging, e.g. in space, due to the effects of time delay caused by the large distances. The attention from telesurgery in space shifted to shorter distance telesurgery solutions, leading to the introduction of the first commercial oriented surgical robots into the market by the end of the 1980 s.

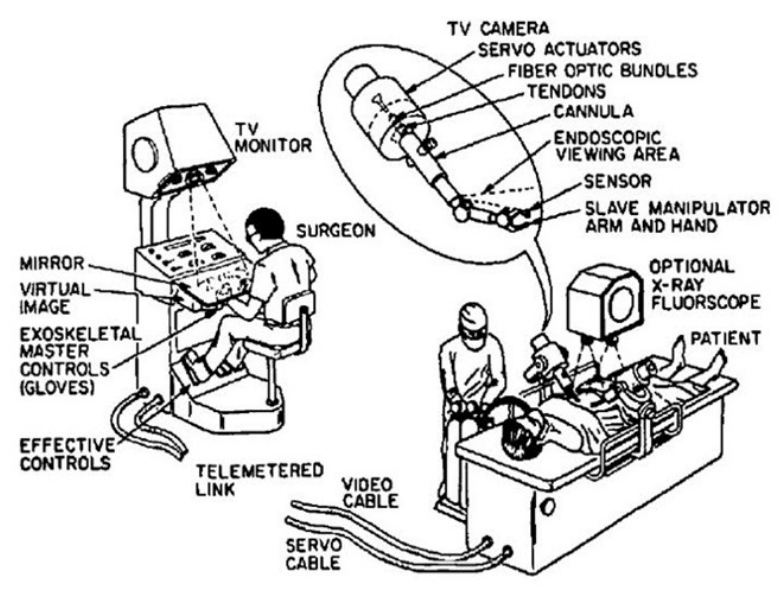

Figure 1

The first concept of a telesurgical robotic system from a USA project proposal (1971) [6].

The first telesurgical systems were designed to improve the dexterity of the surgeons, to increase the reliability of the surgical interventions and to improve the accuracy of the manipulations. Academic centers started to get involved in the development of new systems around the world in the 1980s. The first robot that was explicitly used and designed for patient treatment assistance was the Arthrobot of 
Hearthrob, in 1983, together with a scrub nurse robot. The development was led by J. McEwen, G. Auchinlec and B. Day at the University of British Columbia, Canada [7]. The first procedure, assisted by that robot was carried out in 1984 at the UBC Hospital, organized by the same academic institution. A year later, more than 60 arthroscopic procedures were documented [8], however, the submitted patent referred it to as an active supporting device rather than a treatment device [9]. The developer group from UBC submitted another patent on their newly developed robot the same year, but the submission was withdrawn six years later [10]. Nevertheless, they introduced an arm-holder version of the robot, intended for in-surgery use [11].

Thirty years ago, a robot was used on a human patient for the first time, providing direct surgical support. It was proven that accurate stereotaxis could be achieved by the use of robotic surgical systems, in a CT-guided brain biopsy setup shown in Fig. 2.
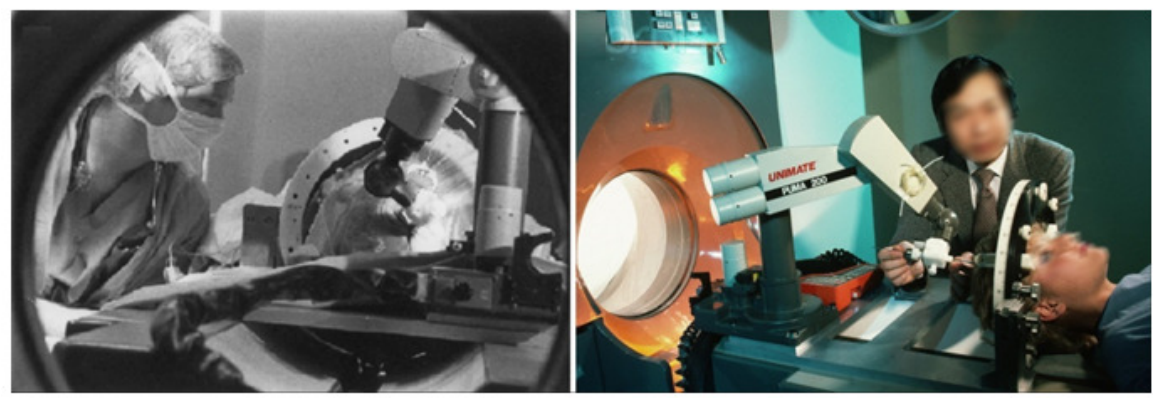

Figure 2

Puma 200, the first robot used for assisting human neurosurgery (1985) [12].

The Puma 200 (Programmable Universal Machine for Assembly) was used for holding and manipulating a biopsy cannulae, navigated by a stereotactic frame mounted on the base of the robot. The intervention was carried out under the supervision of Y. S. Kwoh, at the Memorial Medical Center in Long Beach, CA, USA [12]. The Puma 200 provided a development platform for many novel instruments in the later years. It was used for image processing, performing complete neurosurgical operations, all of which was achieved by manipulating probes during the positioning of the robotic arm.

The U.S. Army has invested a lot of resources into robotic surgery ever since the beginning. It was evident that robotic telesurgery would allow medical assistance and treatment to the soldiers on the battlefield, increasing safety and decreasing treatment costs. Early prototypes of remote healthcare systems were introduced by the Telemedicine \& Advanced Technology Research Center (TATRC), based on the concept of telesurgery proposed by NASA. The Green telepresence system is considered to be the first system where doctors could apply treatments to patients from a distance, introduced in 1991 [13]. This system can be considered as the predecessor of the later most successful da Vinci surgical system. The Green telepresence 
system was a prototype for telesurgery, but from the structural and functional design point of view, it already contained the fundamental concept for today's teleoperation systems. Even more, it incorporated force feedback, which is not yet part of the commercial systems today. The very first system used for long distance experiments was created by the Jet Propulsion Laboratory (Pasadena, CA) and used for experiments reaching Milan, Italy (1993) [14]. Several years later, the U.S. Department of Defense launched it's long distance medical assistance project, the Trauma Pod. The project goal was to demonstrate the feasibility of an integrated, robot-driven emergency care unit, planned to be used by combat surgeons in order to perform remote operations by $2025[15,16]$.

\section{Early Prototypes and System Concepts}

The real growth of surgical robotics happened in the 1990s, in which Computer Motion Co. (Goleta, CA) played and important role. Computer Motion was founded in 1989, and they were specialized in developing systems for laparoscopic surgery. Their first product, the AESOP (Automated Endoscopic System for Optimal Positioning) was an endoscopic camera holder, used for camera assistance [23]. Nonetheless, this was the first tool of its kind to be approved by the Food and Drug Administration (FDA). The second generation of AESOP was capable of voice control and was introduced in 1996, while the third generation, released two years later was improved by adding an additional Degree(s) of Freedom to the robotic arm, and further developed for networking with other devices in the operating room. The AESOP arms were later extended, making them capable of controlling a wide range of surgical instruments. The new system was named Zeus Robotic Surgical System, which included three arms [25].

In the year of 1995, the first prototype was created, while the first animal trials were conducted in 1996 (tubal re-anastomosis). Two years later, the first coronary artery bypass graft (CABG) was carried out, and the system was improved with new components through the early years of the 2000s, such as the Micro-Wrist and the Micro-Joint. The latter was improved to hold and manipulate more than two dozens of different surgical instruments, including scissors, dissectors, scalpels and hooks. FDA approval of the Zeus system came in 2001, which opened up new frontiers in clinical use. The high-end Zeus systems were capable of filtering hand tremor, increasing the reliability of the interventions. The system was designed for Minimally Invasive Surgery (MIS), making it an appropriate tool for beating heart surgery, mitral valve surgery or endoscopic CABG.

Imperial College (London, UK) has been a European flagship institute of surgical robotics since the mid 1980s, becoming a lead research center for prostate surgery [28]. They developed the system called PROBOT for transurethral resection of the prostate (TURP) procedures. This procedure is usually carried out manually with instruments inserted into the urethra and reaching the prostate gland from the inside. Then a wire cutter is inserted, and a conic shape is excised from the prostate. This is often performed manually, however the procedure is long and the absorp- 


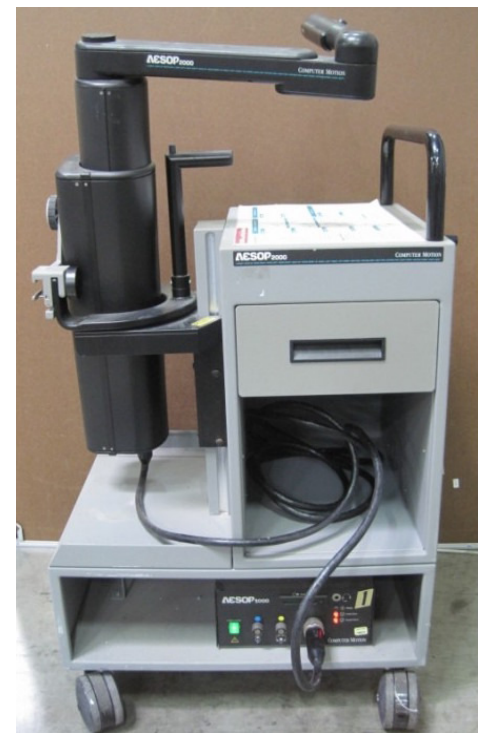

Figure 3

The AESOP surgical robotic platform for camera handling.

tion of hypo-osmolar irrigation fluid can cause further complications. The first five human patients were treated between 1991 and 1993. The successful beginning led to the establishment of the a new Science Engineering Research Council funding initiative. However, the activities at Imperial College were not limited to urologic procedures. They also developed the ACROBOT system to precisely shape the bone surface of the knee for perfect alignment of implants. This system was used to register the surgeon's movements and restrict them to safe regions (active constraints).

Integrated Surgical Systems (ISS, Santa Monica, CA) created the first orthopedic surgical system in 1992, after six years of development at the IBM T. J. Watson Center and U.C. Davis [25]. ROBODOC became quite popular, being the first robot to be used in robot-assisted human hip replacement. It was rebranded to Curexo Robodoc in 2007 and to THINK Surgical in 2014. ISS used to lead the research and development of neurosurgical robotic devices as well. Their robot called NeuroMate-a stereotaxic targeting device for neurosurgery- was the first neurosurgical robot to get FDA clearance. Neuromate was originally designed based on an industrial robot, and it was developed market-ready by Innovative Medical Machines International, Lyon, France. It successfully used image guidance based on preoperative 3D medical images such as Computed Tomography (CT) or Magnetic Resonance Imaging (MR). In the beginning of each operation the robot held a calibration cage and X-ray imaging was used to register the patient's coordinate system to the robot. After the registration, a PC controlled the robot based on the preoperative plan. The robot could take advantage of movements with $5 \mathrm{DoF}$. When the robot reached position, the neurosurgeon inserted the tool through the robot's guiding channel. With $\pm 1 \mathrm{~mm}$ accuracy, the NeuroMate robot enabled more accurate targeting in neurosurgery and therefore the surgeons could reach areas where 
operation had been impossible before.

The first surgical robot for cardiac procedures was the ARTEMIS system. It was designed as a teleoperation and telepresence system for cardiac procedures. Since it was used for training, planning and executing different minimally invasive procedures was part of the design. ARTEMIS had two master-slave units and an endoscope guiding system. The slave side was designed in the way that the manipulators were pivoting around a predefined point aligned with the thoracic insertion point. After the initial setup, the surgeon was able to operate with 6 DoF instruments from the master console. The system also incorporated a 3D endoscopic vision system, which was operated from an additional joystick, separately from the other master manipulators [21].

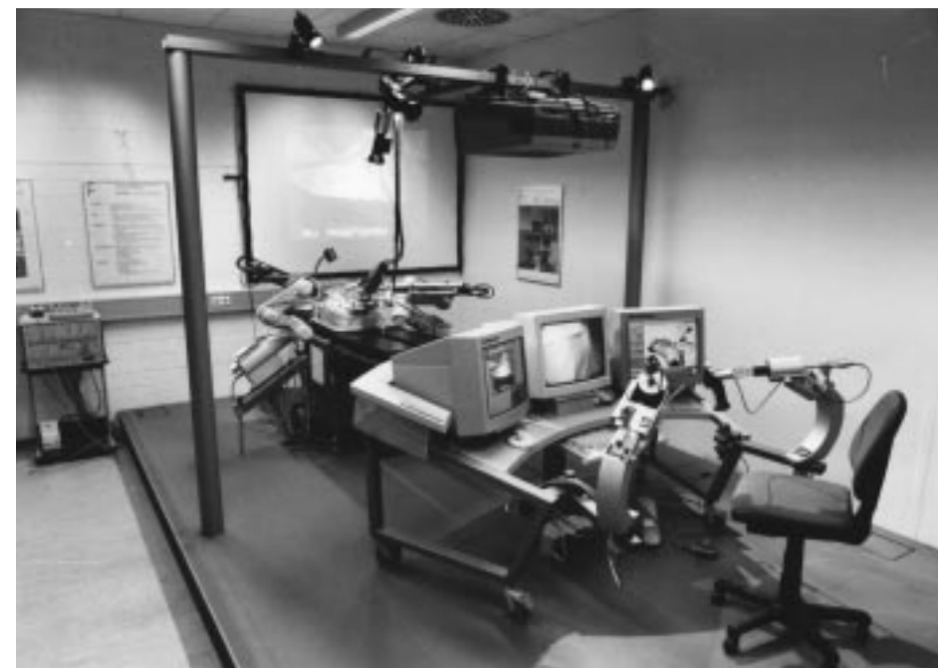

Figure 4

The Artemis surgical robot [22].

After the groundbreaking work was done by these robots, other systems started to appear on the market, targeting specific fields undiscovered by previous solutions. Over 500 research projects were submitted leading to dosens of system prototypes [1]. Many of these systems were discontinued, and today more than $80 \%$ of the market is owned by Intuitive Surgical's da Vinci.

\subsection{The Concept of Robotic Assisted Microsurgery}

The concept of Robotic Assisted Microsurgery (RAMS) plays an important role in the design and development of robotic surgical devices. Originally coming from the NASA laboratories, it appeared as a side-innovation for telerobotics and microsurgery. It was S. Charles who first proposed the idea of using telerobotic systems as a tool in microsurgical procedures [17]. The project was initiated at the NASA JPL, more precisely within the Advanced Teleoperation Lab (ATOP), which, at that 
time, was directed by A. Bejczy. RAMS was developed around 1994, inheriting the basic concepts of the NASA telesurgical technologies (Fig. 5).

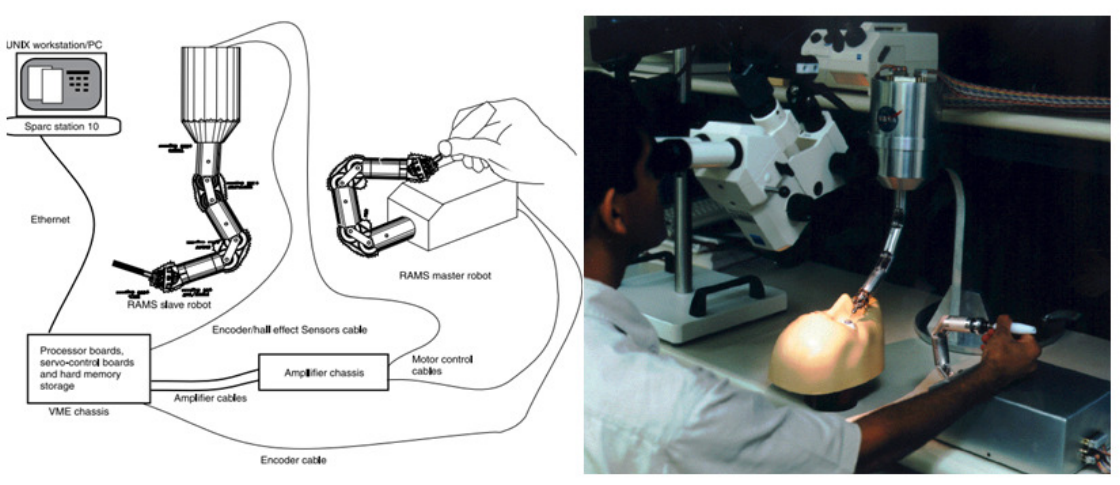

Figure 5

The concept and realization of the RAMS system at NASA JPL ATOP [18, 23].

The developed robot was built up from a 3-axis wrist that was mounted on a torsoshoulder-elbow assembly, capable of 6 DoF motions. The robot included a manipulator, whereas the engineers were aiming to create similar kinematic layout for both master and slave sides of the instrument. The use of RAMS instruments was later extended for carrying out coronary artery anastomoses [18], and soon successful animal trials were documented. Other studies pointed out that robotic devices could also be used for endoscopic cardiac surgical procedures, including endoscopic anastomosis $[19,20]$.

\section{Basic concepts in Computer-Integrated-Surgery}

With the spread of surgical robotics initiatives, a new domain was established. CIS was born based on the understanding that engineering can provide surgeons with tools, making procedures safer, less invasive and eventually more efficient. Arguably, the appearance of digital medical imaging brought the biggest impact, when it finally replaced the previously used analog technology. Not only did it provide the capability to do fast intra-operative scans, but also made pre-operative imaging more informative. One great example of this is the appearance of CT devices. Before CT was used, surgeons only had access to projective X-ray images; with CT, a clear 2D slice of the human body could be obtained, and even the 3D reconstructions of the anatomy became achievable. From computer-based imaging and other fields such as genetics, blood testing, etc., we can generate a database of patient specific information, which was previously inaccessible. This patient specific information made it possible for CIS to improve, and fundamentally change treatment planning. Without accurate imaging, it was only possible to guess the underlying anatomy with physical examination, and often surgeons needed to face unexpected 
situations after the operation started. Today, procedures can be planned on accurate, patient specific data gained from imaging (Fluoroscope, US, or even MR) and other modalities. This enables surgeons to decide where and when to operate, and to provide the best possible quality of life for the patient as a result of the surgery. As devices became more portable, it was possible to move them into the Operating Room (OR), where they could be used for intra-operative imaging, or to provide assistance to the procedure. Data collected from these devices can be used to adjust or re-plan the procedure during the execution. Finally, after the procedure, the data collected could be used for statistical analysis, which could provide important information on performance and become a basis of further development. This loop of Computer-Integrated-Surgery is shown on Fig.6. Pretty much all of the concept robots listed above choose one or another approach to optimize its use within the frames of CIS. Over the years three, distinct strategies prevailed, introduced in the following.

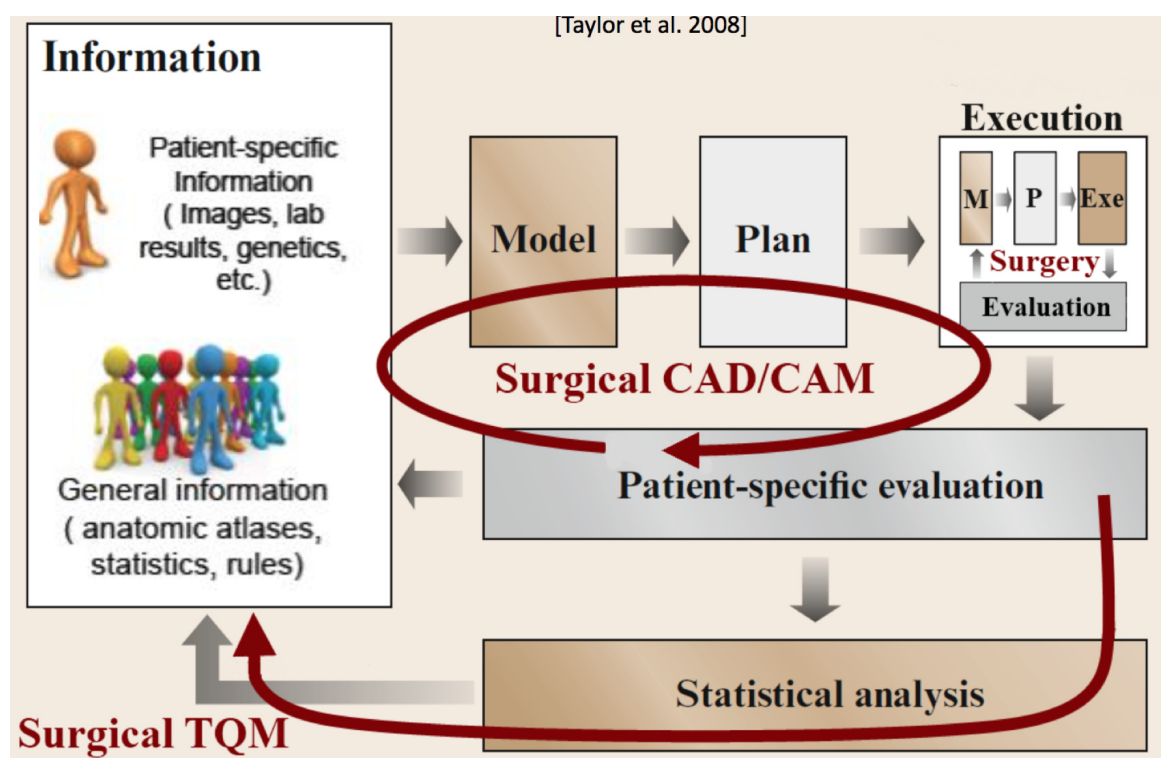

Figure 6

Basic concept of Computer-Integrated-Surgery [27].

\subsection{Types of Robotic Assisted Surgery}

Robotics can assist surgeons in various ways, and it is probably an impossible task to find a uniform characteristic for all cases besides that engineering work enhances the performance of the surgeon. In the following, we present the three main approaches surgical robotics follows nowadays giving an example for each one trough a robotic platform used in surgical practice. 


\subsubsection{Robotic telesurgery (Human-in-the-loop control)}

Robotic telesurgery devices are applications where the surgeon remotely controls the robot performing the operation (teleoperation). The da Vinci system-which is definitely the most successful robotic platform in existence-follows this approach. The surgeon works at a console, his/her hand movements are perceived by the master manipulator, and transmitted onto the patient side manipulator, holding the actual tool, where the fingers (thumb and index) are mapped to the tip of the tools. This has a major ergonomic benefit, since statistics have shown that the incidence of chronic back pain increased significantly among surgeons performing conventional laparoscopic procedures, which is due to the fact that during these procedures, the surgeon stands next to the patient and forced to maintain an anatomically inadequate position. Letting the surgeon work at a specially designed workstation provides a great opportunity for engineering an ergonomic environment, lowering the physical and mental stress on the surgeon. Applications based on teleoperation methodology can also incorporate functions to cancel out the surgeons tremor, or to provide magnification to the view of the field the surgeon is interested in. However, these platforms do not incorporate much automation, as the feedback loop of the control requires the continuous involvement of the human operator. This is also a benefit for the manufacturer from the regulatory point of view, since it was easier to convince the authorities about the systems' safety, while the human surgeon is always in charge of the robot's motion. Teleoperation would be a preferred way to do interventions in remote areas where specialists are not available, or could not reach the patient in time. Extreme environment research such as a space expeditions or military applications are driving this field, as they face the same difficulties. In these situations teleoperation would be required to operate with large physical distances between the master and slave sides, and time delay would become a significant issue, probably compromising the success of the surgery. Several algorithms were proposed for the control of these systems, but there is no clear solution for the problem yet [26]. The main reason behind this is that when time delay becomes noticeable, even if the system stays stable, the operation becomes harder and takes significant processing time to complete. Time delay can be shortened by using dedicated channels, however these are costly, and therefore not applicable beyond some special cases. Another solution is to model the behavior of the tissue on the master side, and later correct the model based on the data received from the patient side sensors. Unfortunately the complexity of the operating field can not be sufficiently modelled today.

\section{The da Vinci Surgical System}

Intuitive Surgical Inc. is the manufacturer of the da Vinci Surgical System, which was created for MIS procedures. The da Vinci was the first telerobotic system for human use that was cleared by the Food and Drug Administration (FDA) in 2000. Approval of the system included urological, thoracoscopic, gynecological and laparoscopic procedures, both for adult and pediatric use. The prototype Mona was involved in the first trials in 1997, while the first closed-chest, multi-vessel cardiac bypass procedure was performed using this robot in 1999. Originally, the system was designed for carrying out cardiac procedures, such as beating heart surgery, yet the real popularity of the system was brought by the rapid adaptation in radi- 

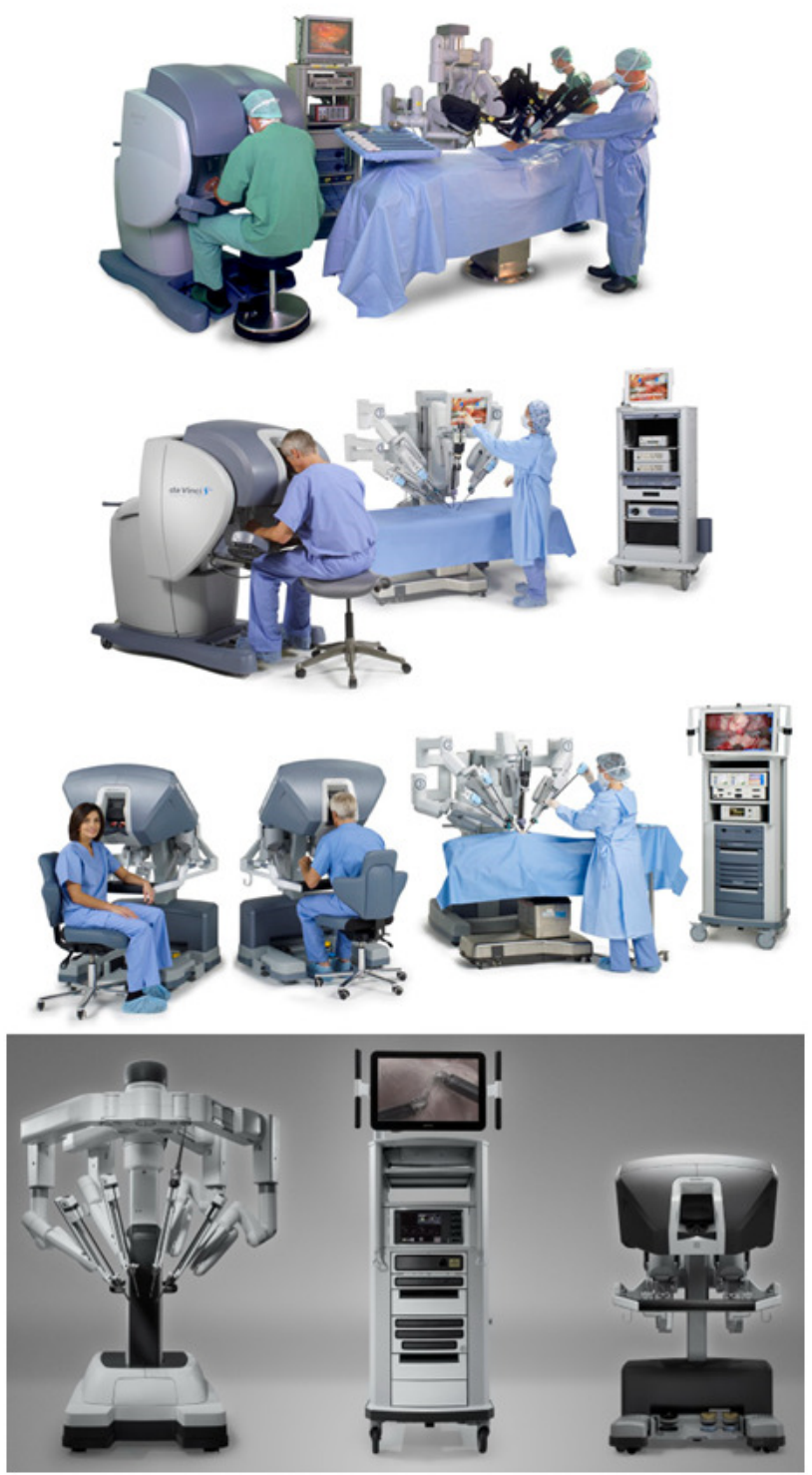

Figure 7

Generations of the da Vinci Surgical System from 1999 to 2014. Da Vinci Classic, S, Si and Xi systems. Image credit. Intuitive Surgical Inc.

cal prostatectomy and hysterectomy procedures. The recent growth of trans-oral robotic surgery has also brought new prospects to the development of the da Vinci system.

The da Vinci is a typical master-slave system, consisting of a master side console, a patient side set of robotic arms and a visualizing system. The basic architecture 
has remained the same, following the initial concepts from the first generation. The four generations of the da Vinci are the Classic, S, Si and the Xi, as shown in Fig. 7. The fundamental concept has not changed since the NASA's '70s plans, while the hardware and software capabilities have improved dramatically.

The master side manipulators are serving as the interface for the surgeon, allowing $\mathrm{him} /$ her to manipulate the tools virtually. A 3D display system is also integrated at the master side, showing the surgical field recorded by the stereo camera endoscope. The connection of two master consoles is also possible enabling efficient training and teaching. In all generations, the maximum number of patient side manipulators is 3 , extended with an additional robotic arm holding the endoscopic camera. The arms are copying the movements of the surgeon's arms, following them in real-time. This can only be achieved if the surgeon is in the same room with the patient in order to keep the latency (time delay) low. To achieve this, Intuitive used the technology of pre-existing systems, which is supported by the fact that after its incorporation, it closely observed the RAMS patents for force reflection surgery from the California Institute of Technology. Later one of the first da Vinci was installed at one of the NASA facilities. In the same year, the first closed-chest beating heart cardiac bypass surgery was performed using the Zeus robot, the great competitor at that time.

\subsubsection{Image-Guided Surgical Robotics}

Medical imaging went trough tremendous improvements when it moved from ana$\log$ technologies to information based methods. Having accurate digital images of the patients opened up new possibilities in image guidance, allowing more accurate targeting in fields like oncology or neurosurgery. Unlike telesurgery, the imageguided approach typically incorporates autonomous execution of the whole or part of the surgical plan, for which the machine does not require a human operator in the control loop. Real-time intra-operative tracking of the tools and the patient, achieved with either 3D cameras, electromagnetic trackers or other modalities [29]. Accurate navigation can only be achieved if the reference image is registered to the physical world and the transformations between the different coordinate frames are known. The most commonly used approach is the rigid frame registration, registration for distorted images is achieved by numeric approximation. The ROBODOC system is (cleared by the FDA for total knee and hip replacement surgeries) a typical example. It uses pre-surgical 3D planning based on the patients CT scan. Given the surgical plan, the ROBODOC is able to execute the bone milling task with submillimeter accuracy. In this application the robot is actively milling and the surgeon only provides supervision to the procedure.

\subsubsection{Cooperative or Hands-on Surgical Robotics}

The human hand is a remarkable multi-purpose grasping tool with amazing capabilities in terms of dexterity. This is why surgeons tend to prefer using it during the surgery, rather than just relying on some limited human-machine interfaces. Another argument against the telesurgical approach is typically the loss of certain 


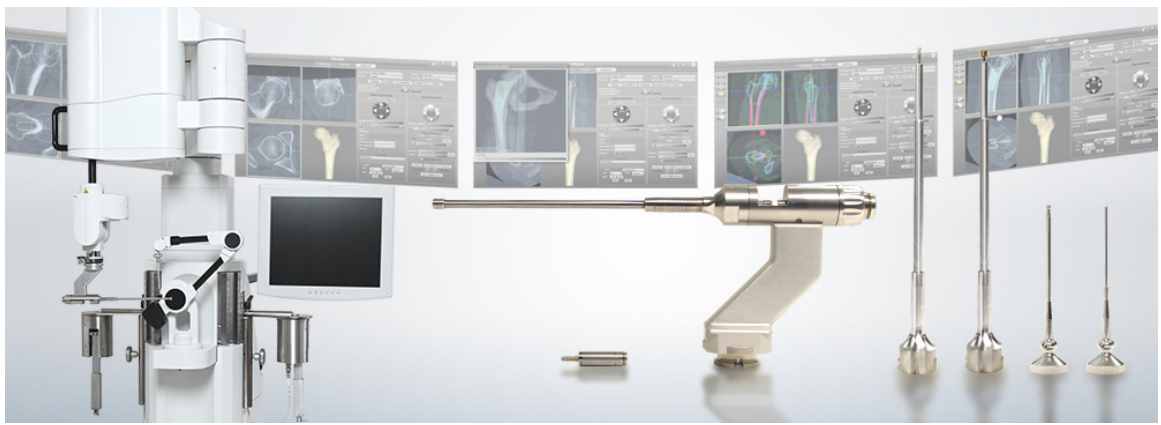

Figure 8

The THINK Surgical system from Curexo Technology Corp. Photo courtesy by Curexo Technology

Corp.

sensory feedback from the operational site. In the meanwhile, for some special surgical tasks, more sensitivity or a wider range of motions are required, beyond that of our physical construction can provide. Cooperative control offers a plausible alternative, enabling the surgeon to use a robotic tool as a slave device right at the spot of the intervention, directly controlling it with its hands. This is typically achieved with force/torque sensors and control [32]. Eye surgeons face this problem in their everyday work. During a procedure it is required to access areas only a couple of cell layers thick, with a resistance impossible to feel, when the procedure is performed manually. During these tasks, ophthalmologists are usually left to use visual feedback only. The Steady Hand device (Fig. 9.), developed at the Johns Hopkins University (Baltimore MD, USA) is intended to tackle this problem. It is a cooperatively controlled robot, where the surgeon moves the tool attached to the robotic platform by exerting forces on it. The Steady Hand robot is able to cancel out some of the tremor and unwanted motions from the hand movement. It also incorporates sensitive pressure sensors for measuring the small changes in tissue resistance. By amplifying these forces, it can give a sensible feeling to microsurgery which did not exist during traditional procedures. It can also provide visual guidance and audible sounds to better assist the surgeon.

Bordering image-guided and cooperative control paradigm, the RIO system from MAKO (acquired by Stryker in 2015). It uses image guidance to control the milling tool mounted at the end of a robotic arm, which is eventually mounted on a movable cart. Unlike the ROBODOC, the RIO system does not carry out the actual milling, but instead it creates resistance on predefined boundaries-also called virtual fixtures-allowing the surgeon to access only a restricted area of the operation field. With this mechanism, the RIO system can protect from unwanted errors, but, in the case of a registration, planning or system error, the surgeon can interfere as he/she is still in complete control of the instrument. 

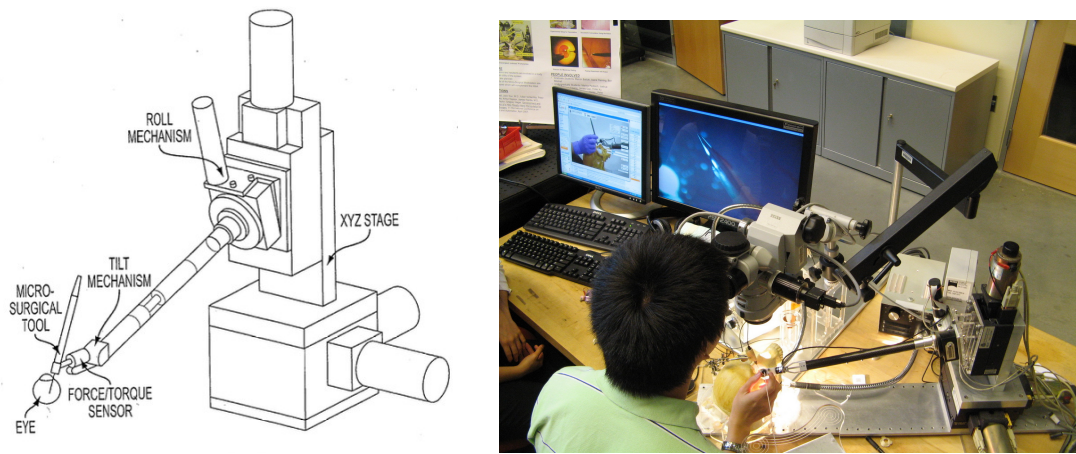

Figure 9

The schematic view of the Steady Hand device, and the experimental setup at the Johns Hopkins University.

\section{Surgical Robotics in Extreme Environments}

DARPA and NASA remained deeply interested in telerobotic surgery throughout the past decades. Surgical procedures in space, as it was pointed out in the early trials by NASA, carry many difficulties and challenges, and the signal latency is one of the many problems to be solved [33]. In order to investigate the possibilities of operation in weightlessness, a Soviet team carried out the first animal surgery of this kind during a parabolic flight in 1967. Thirty years later, an experiment was performed on a rat model during the STS 90 Neurolab Mission, however, the abdominal cavities were not treated in this procedure. The first zero gravity surgical experiment by NASA was a suturing task performed by an M7 robot (developed by SRI, Sacramento, CA), during hyperbolic flight. The task was a classical, teleoperated knot tying, where the slave and master sides were equipped with acceleration compensation. The inconclusive results showed that human surgeons were better at adaptation than the robots, in the case of extreme conditions.

The European Space Agency (ESA) took part in the first European initiative for investigating surgical procedures in weightlessness on board of a Zero $G$ plane in 2003. In 2006, a cyst was removed from a patient's arm during parabolic flight. The further planned projects for telesurgery experiments during parabolic flights have been postponed ever since.

When a procedure in space is taken place beyond Earth's orbit, numerous difficulties arise with telesurgical robotics. Theoretically, communication between the master and the slave side is propagating at the speed of light. However, this speed already causes a latency of more than 1 second on the Earth-Moon distance. Inter-planetary distances, such as the Earth-Mars relation increases the delay to 44 seconds, which can become even more because of the significant computational efforts in compressing and decompressing video data.

Extreme telesurgery experiments under terrestrial conditions have long been investigated by NASA. Experiments were primarily carried out during the NASA Extreme 
Environment Mission Operations (NEEMO). This is the world's only permanent undersea laboratory, near Florida Keys, 19 meters below the water surface. State-ofthe-art computers and equipments are used by astronauts, biologists and engineers, with the intention of finding the consequences of operating in extreme environments, simulating the tasks of space missions.

The Zeus robot was involved in the 2004 series of experiments, during the $7^{\text {th }}$ NEEMO project, focusing on telementoring and teleoperation. There were five test conditions investigated: ultrasound-guided abscess damage, ultrasonic examination of abdominal organs, cystoscopy, laparoscopic cholecystectomy and renal stone removal. Two astronauts, a physician and a surgeon were involved in this undersea experiment, while the robot was controlled remotely from the Centre for Minimal Access Surgery, London, UK. The distance was 2500 kilometers, and the latency was manually increased to a value between 100 and 2000 milliseconds. Results showed that the crew members with less or no expertise were quite successful in performing the interventions based on the guidance of the telementors. The effectiveness of teleoperation and telementoring was compared, and an important deduction was made: teleoperation procedures were more accurate, but took significantly more time and effort than telementoring.

In 2006, during the $9^{\text {th }}$ NEEMO project, an assembly task was given to the crew for installing an M7 surgical robot for performing abdominal surgery in a virtual environment. The latency was set to 3 seconds. As the intention was to replicate a Lunar environment, the robot was intended for manipulating rock samples from the bottom of the ocean. Telementoring tasks were also included, focusing on fatigue and other stressor effects on the performance of the crew.

The $12^{\text {th }}$ NEEMO project investigated the feasibility of telesurgery using the Raven and $\mathrm{M} 7$ robots, as shown in Fig. 10. The structure of the crew was the same as in the $7^{\text {th }}$ project, performing suturing tasks, measuring the effectiveness of teleoperating surgeons located in Seattle, Washington. Commercial internet connection was used, with a relatively short latency time.

The NEEMO projects conclusion stated that the high quality tactile and visual feedback are equally important in order to create reliable teleoperation environments. High quality video streaming, however, is difficult to achieve due to the limited bandwidth. The bandwidth of $50 \mathrm{Mbps}$ is already available on the International Space Station, although Mars missions are still calculated with the half of this amount by 2025 .

\section{Discussion \& Conclusion}

Surgical robotics originated from the need to operate over long distances, where a medical doctor could not get physical access to the patient. This requirement emerged first at NASA missions for space expeditions, but was quickly picked up by the military as well, where DARPA funded the key research projects on telesurgical robotics. The most successful robotic system for surgery, the da Vinci grew out of these early results in 1998, successfully combining the advantages of various prototypes. Since then, technology continued to improve, and instead of the military applications, the private sector has become the driving force of the surgical 

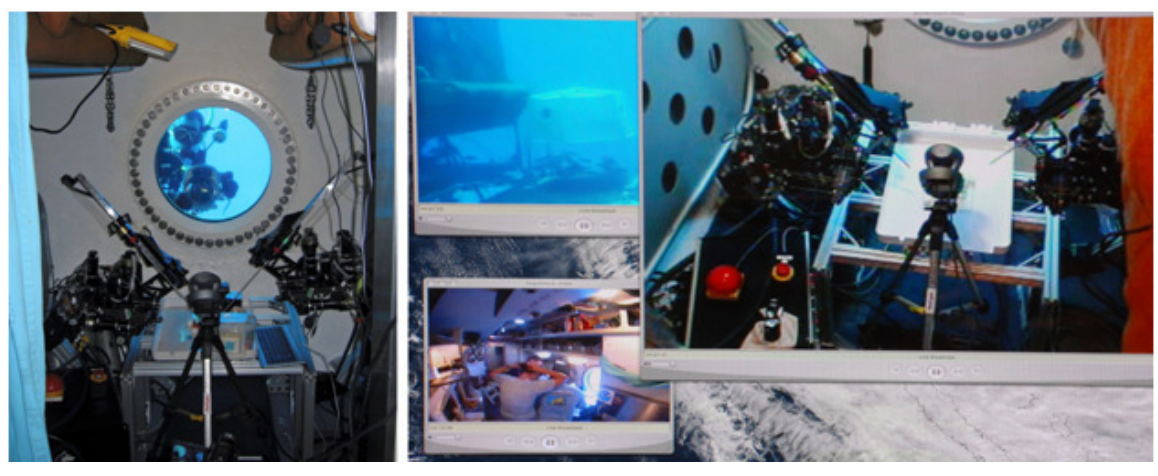

Figure 10

Underwater experiments at NASA Aquarius station during the NEEMO 12 project: setup and remote view of the Raven robots, developed at the UNiversity of Washington (Seattle, WA). Photo credit:

NASA.

robotics industry, which is estimated to be around $\$ 5$ billion per year. Along with the constant improvement in telesurgery, other robotic devices appeared for enhancing surgical capabilities. Three major approaches can be defined within the field of Computer-Integrated Surgery, these are telesurgery, image-guided surgery and cooperatively controlled surgical robotics. In all of these three fields, an important trend is that pre- and intra-operative information-in the form of imaging, physiological data collection, etc.- - are playing an increasingly significant role during the procedure, and are enabling robotic systems to gain more autonomy. The field is rapidly changing thanks to the hundreds of research teams focusing on relevant projects, and nowadays, major players, like Google are also entering the arena. The computing capabilities of modern ICT devices are to allow the usage of more sophisticated systems, however, overarching regulations and standards in the field are still missing. Surgical robotic specific standards currently under development will make it possible for the industrial players to better design their systems, to be able to prove their safety and accuracy to the authorities. As for today, most applications keep the human operator active in the control loop, enabling the robot only to enhance the surgeons' capabilities. Autonomous task execution on Earth and in space remains a topic of the future.

\section{Acknowledgement}

Tamás Haidegger is a Bolyai Fellow of the Hungarian Academy of Sciences. This work has been supported by ACMIT (Austrian Center for Medical Innovation and Technology), which is funded within the scope of the COMET (Competence Centers for Excellent Technologies) program of the Austrian Government. 


\section{References}

[1] M. Hoeckelmann, I. J. Rudas, P. Fiorini, F. Kirchner and T. Haidegger, "Current Capabilities and Development Potential in Surgical Robotics" International Journal of Advanced Robotic Systems, vol.12(61) pp. 1-39., 2015

[2] Á. Takács, L. Kovács, I. J. Rudas, R. E. Precup and T. Haidegger, "Models for Force Control in Telesurgical Robot Systems" Acta Polytechnica Hungarica, vol.12, issue 8, pp. 95-114., 2016

[3] C. R. Doarn, A. E. Nicogossian and R. C. Merrell, "Applications of telemedicine in the United States space program" Telemed J, vol. 4, no. 1, pp. 19-30, 1998.

[4] K. Corker, J. H. Lyman and S. Sheredos, "A preliminary evaluation of remote medical manipulators" Bull Prosthet Res, issue 16, vol 2. pp. 107-134, 1979.

[5] A. D. Alexander, "Impacts of Telemation on Modern Society" in On Theory and Practice of Robots and Manipulators, Springer Berlin Heidelberg, 1972, pp. 121-136.

[6] A. D. Alexander, "Impacts of telemation on modern society," Jan. 1973.

[7] Wikipedia: Robot-assisted Surgery

[8] O. Lechky, "Worlds first surgical robot in B.C.," The Medical Post, vol. 21, no. 23, pp. 92-93, Nov. 1985. Available: http://www.brianday.ca/ imagez/1051_28738.pdf

[9] Powered surgical retractor. United States Patent 5271384. Available: http: //www.freepatentsonline.com/5271384.html Accessed: Nov. 4. 2015

[10] Medizinischer Roboter, EP Patent App. EP19,860,106,362, https: //www . google.com/patents/EP0201883A2?cl=de, 1986, Accessed: Nov. 5. 2015

[11] Patient limb positioning apparatus, US Patent US4807618. Available: http: //www.google.com/patents/US4807618 Accessed: Nov. 5. 2015

[12] Y. S. Kwoh, J. Hou, E. A. Jonckheere and S. Hayati, "A robot with improved absolute positioning accuracy for CT guided stereotactic brain surgery," IEEE Trans. on Biomedical Engineering vol. 35, no. 2, pp. 153-160, Feb. 1988.

[13] J. C. Bowersox, A. Shah, J. Jensen, J. Hill, P. R. Cordts and P. S. Green, "Vascular applications of telepresence surgery: initial feasibility studies in swine," Journal of Vascular Surgery, vol. 23, no. 2, pp. 281-287, Feb. 1996.

[14] A. Rovetta, R. Sala, F. Cosmi, X. Wen, S. Milanesi, D. Sabbadini, A. Togno, L. Angelini and A. K. Bejczy, "A New Telerobotic Application: Remote Laparoscopic Surgery Using Satellites and Optical Fiber Networks for Data Exchange" The Intl. J. of Robotics Research vol. 15, no. 3, pp. 267-279, Jun. 1996. 
[15] R. M. Satava, "Virtual reality, telesurgery, and the new world order of medicine," Journal of Image Guided Surgery, vol. 1, no. 1, pp. 12-16, 1995.

[16] P. Garcia, J. Rosen, C. Kapoor, M. Noakes, G. Elbert, M. Treat, T. Ganous, M. Hanson, J. Manak, C. Hasser, D. Rohler and R. Satava, "Trauma Pod: a semi-automated telerobotic surgical system," Int. J. Med. Robotics Comput. Assist. Surg., vol. 5, no. 2, pp. 136-146, 2009.

[17] H. Das, T. Ohm, C. Boswell, R. Steele and G. Rodriguez, "RobotAssisted Microsurgery Development at JPL," in Information Technologies in Medicine, M. Akay and Andyrsh, Eds. John Wiley \& Sons Inc., 2001, pp. 85-99.

[18] E. R. Stephenson, S. Sankholkar, C. T. Ducko and R. J. Damiano, "Robotically assisted microsurgery for endoscopic coronary artery bypass grafting," The Annals of Thoracic Surgery, vol. 66, no. 3, pp. 1064-1067, Sep. 1998.

[19] P. D. Le Roux, H. Das, S. Esquenazi and P. J. Kelly, "Robot-assisted microsurgery: a feasibility study in the rat," Neurosurgery, vol. 48, no. 3, pp. 584-589, Mar. 2001.

[20] J. Schiff, P. S. Li and M. Goldstein, "Robotic microsurgical vasovasostomy and vasoepididymostomy: a prospective randomized study in a rat model," Journal of Urology, vol. 171, no. 4, pp. 1720-1725, Apr. 2004.

[21] M.O. Schurr, A. Arezzo, G.F. Buess, "Robotics and systems technology for advanced endoscopic procedures: experiences in general surgery," European Journal Cardio-Thoracic Surgery, vol. 16, Suppl 2, pp. 97-105, 1999.

[22] H. Rininsland, "ARTEMIS. A telemanipulator for cardiac surgery," European Journal Cardio-Thoracic Surgery, vol. 16, Suppl 2, pp. S106-S111, 1999.

[23] S. Saraf, "Role of robot assisted microsurgery in Plastic Surgery," Indian Journal of Plastic Surgery, vol. 39, no. 1, pp. 57-61, 2006.

[24] S. E. Butner and M. Ghodoussi, "Transforming a Surgical Robot for Human Telesurgery," IEEE Trans. on Robotics and Automation, vol. 19, no. 5, pp. 818-825, 2003.

[25] J. Rassweilera, J. Binderc and T. Frede, "Robotic and telesurgery: will they change our future?," Current Opinion in Urology, vol. 11, pp. 309-320, 2001.

[26] P. F. Hokayem and M. W. Spong, "Bilateral teleoperation: An historical survey," Automatica, vol. 42, no. 12, pp. 2035-2057, 2006.

[27] R. H. Taylor, A. Menciassi, G. Fichtinger and P. Dario, Medical robots and systems. In: B. Siciliano, O. Khatib, eds., Springer Handbook of Robotics. ch. 52 Berlin: Springer-Verlag, 2008.

[28] S.J. Harris, F. Arambula-Cosio, Q. Mei, R.D. Hibberd, B.L. Davies, J.E. Wickham, M.S. Nathan and B. Kundu, "The Probot—an active robot for prostate resection.”, Proc. Inst. Mech. Eng. H., vol. 211(4), pp. 317-325., 1997. 
[29] Y. Lu, C. Li, M. Liu, J. Fritz, J. A. Carrino, L. Wu and B. Zhao, "MRIguided stereotactic aspiration of brain abscesses by use of an optical tracking navigation system," Acta Radiol, vol. 55, no. 1, pp. 121-128, 2014.

[30] A. P. Schulz, K. Seide, C. Queitsch, A. von Haugwitz, J. Meiners, B. Kienast, M. Tarabolsi, M. Kammal and C. Jrgens, "Results of total hip replacement using the Robodoc surgical assistant system: clinical outcome and evaluation of complications for 97 procedures," The International Journal of Medical Robotics and Computer Assisted Surgery, vol. 3, no. 4, pp. 301-306, 2007.

[31] A. N. Supe, G. V. Kulkarni, P. A. Supe, "Ergonomics in laparoscopic surgery," Journal of Minimal Access Surgery; vol.6(2) pp. 31-36, 2010

[32] T. Haidegger, B. Benyó, L. Kovács and Z. Benyó, "Force Sensing and Force Control for Surgical Robots," Proc. of the 7th IFAC Symposium on Modelling and Control in Biomedical Systems, Aalborg, Denmark, Aug. 12-14, pp. 413-418, 2009

[33] T. Haidegger, J. Sándor and Z. Benyó, "Surgery in space: The future of robotic telesurgery," Surgical Endoscopy vol. 25(3) pp. 681-690, 2011 